Pacific Journal of Mathematics

D-DIMENSION. I. A NEW TRANSFINITE DIMENSION 


\title{
D-DIMENSION, I. A NEW TRANSFINITE DIMENSION
}

\author{
DAVID W. HENDERSON ${ }^{1}$
}

The large inductive dimension (Ind) can be extended, by transfinite induction, to all the ordinals. The transfinite inductive dimension so obtained has been investigated by many authors. Unfortunately, it does not possess many of the nice properties which are possessed by the inductive dimension in finite-dimensional spaces. For instance, the transfinite inductive dimension fails to be monotone for separable metric spaces; and it fails to satisfy the sum theorem, even for compact metric spaces.

This paper introduces a new transfinite dimension called $D$-dimension, which is defined for all metric spaces. For finitedimensional spaces, $D$-dimension equals Ind. It is shown that $D$-dimension is also a monotone and local property and that it satisfies several sum and product theorems. These properties lead to a characterization of $D$-dimension.

In particular, $D$-dimension as a topological function from all metric spaces to the ordinals ( $\geqq-1$ ), with an extra symbol, $\Delta$, added, satisfies the following axioms: (We use the conventions that, for each ordinal $\alpha, \Delta>\alpha, \Delta+\alpha=\Delta+\Delta=\alpha+\Delta=\alpha \oplus \Delta=\Delta \oplus \Delta=\Delta$, where ' $\oplus$ ' denotes the natural sum of ordinals.)

I. (Finite-Dimensional Spaces). If either $D(X)$ or Ind $(X)$ is finite, then $D(X)=\operatorname{Ind}(X)$.

II. (Local Property). $D(X)=$ l. u.b. $\left\{D_{p}(X) \mid p \in X\right\}$, where $D_{P}(X)=\operatorname{minimum}\{D(N) \mid N$ a neighborhood of $p$ in $X\}$.

III. If $F$ is a closed subset of the space $X$, then $D(X) \leqq$ $D(X-F)+D(F)$.

IV. (Monotone). If $Y$ is a subspace of $X$, then $D(Y) \leqq D(X)$.

V. If there is a point $x \in X$, such that $D_{X}(X)=D(X)$, then $D(X \times I)=D(X)+1 . \quad(I \equiv$ the unit interval. $)$

VI. (Sum Theorem). If $A$ and $B$ are closed subsets of $X$, then $D(A \cup B)=$ maximum $\{D(A), D(B)\}$.

VII. If $A$ is a nonempty closed subset of $X$ and $\gamma$ is a limit ordinal, such that each open set that intersects $A$ contains, for each $\beta<\gamma$, a closed (in $X$ ) subset of $X-A$ with $D$-dimension $\geqq \beta$, then $D(X) \geqq \gamma+$ minimum $\{D(A), \omega\}$.

It is shown that Axioms I, II, III, IV, VII characterize $D$-dimension, and that Axioms I-VI "essentially force" VII. In addition, the following properties are demonstrated: 
VIII. (Product Theorem). $D(X \times Y) \leqq D(X) \oplus D(Y)$.

IX. For each ordinal $\alpha$, there is a space $Q^{\alpha}$ such that $D\left(Q^{\alpha}\right)=$ a. For countable ordinals, these are the compact metric spaces constructed by Smirnov, [9], such that Ind $\left(Q^{\alpha}\right)=\alpha$.

X. Cardinal $(D(X)) \leqq$ weight $(X)$, if $D(X) \neq \Delta$. (The weight $(X)$ is the least cardinal which is the cardinal of a basis for the open sets of $X$.)

With the exception of Axioms I and X, none of these properties are known to be satisfied by Ind.

A second paper will investigate the relation between $D$-dimension and compactifications.

1. Preliminaries. All spaces mentioned in this paper will be assumed to be metrizable spaces.

Definition $(\lambda(\alpha)$ AND $n(\alpha))$. For each ordinal $\alpha$, let $\lambda(\alpha)$ be the largest limit ordinal which is $\leqq \alpha$, and let $n(\alpha)$ be the finite ordinal such that $\alpha=\lambda(\alpha)+n(\alpha)$. We define $\lambda(\Delta)=\Delta$ and $n(\Delta)=0$.

Definition. $(D(X))$. Define $D(\varnothing)=-1$. For a nonempty space, $X, D(X)$ is defined to be the smallest ordinal number $\beta$, if any exist, such that $X=\bigcup\left\{A_{\alpha} \mid 0 \leqq \alpha \leqq \gamma\right\}$, where

(a) each $A_{\alpha}$ is a closed finite-dimensional (Ind) subset of $X$;

(b) for each $\delta, \bigcup\left\{A_{\alpha} \mid \delta \leqq \alpha \leqq \gamma\right\}$ is closed in $X$;

(c) $\lambda(\beta)=\gamma$ and $n(\beta)=$ Ind $\left(A_{\gamma}\right)$, if $A_{\gamma}=\varnothing$ then define $n(\beta)=0$; and

(d) for each $x \in X$, there is a largest $\delta$, such that $x \in A_{i}$. If no such $\beta$ exists then we set $D(X)$ equal to the symbol $\Delta$. If (a), (b), and (c) are satisfied then we will say that $X=\bigcup\left\{A_{\alpha} \mid 0 \leqq \alpha \leqq \gamma\right\}$ is a $\beta$-D-representation of $X$. Also, we shall agree to let $\lambda+(-1)=\lambda$, whenever $\lambda$ is a limit ordinal, so that we can say that the above representation is a $\left(\gamma+\operatorname{Ind}\left(A_{i}\right)\right)$ - $D$-representation. equal.

Theorem 1. If either Ind $(X)$ or $D(X)$ is finite then they are

Proof. If $D(X)=n$ is finite then $X$ has an $n$-D-representation, $X=\bigcup\left\{A_{\alpha} \mid 0 \leqq \alpha \leqq \gamma\right\}$, with $n=\gamma+$ Ind $\left(A_{\gamma}\right)$. Therefore, $\gamma$ must be equal to zero, and thus $D(X)=n=\operatorname{Ind}\left(A_{0}\right)=\operatorname{Ind}(X)$. If Ind $(X)=n$ is finite, then $X=X$ is an $n$-D-representation and therefore $D(X) \leqq n$. Thus $D(X)$ is finite and, by the previous argument, $D(X)=\operatorname{Ind}(X)$.

Theorem 2. If $Y \subset X$, then $D(Y) \leqq D(X)$. 
Proof. If $D(X)=\Delta$, then the theorem holds; therefore we assume that $D(X) \neq \Delta$. If $X=\bigcup\left\{A_{\alpha} \mid 0 \leqq \alpha \leqq \gamma\right\}$ is a $D(X)$ - $D$-representation of $X$, then $Y=\bigcup\left\{A_{\alpha} \cap Y \mid 0 \leqq \alpha \leqq \gamma\right\}$ is a $\left(\gamma+\operatorname{Ind}\left(A_{\gamma} \cap Y\right)\right)$-D-representation of $Y$. Therefore

$$
D(X) \leqq\left(\gamma+\text { Ind }\left(A_{\gamma} \cap Y\right)\right) \leqq\left(\gamma+\text { Ind }\left(A_{\gamma}\right)\right)=D(X) .
$$

2. Sum Theorems. Before proving the two Sum Theorems we prove

LEMMA 1. If $X$ has a $\beta$-D-representation, then there is a $\beta$ - $D$ representation $X=\bigcup\left\{A_{\alpha} \mid 0 \leqq \alpha \leqq \gamma\right\}$, such that Ind $\left(A_{\alpha}\right) \leqq n(\alpha)$.

Proof. Let $X=\bigcup\left\{B_{\alpha} \mid 0 \leqq \alpha \leqq \gamma\right\}$ be any $\beta$ - $D$-representation of $X$. We shall define, inductively, a one-to-one increasing function $f:\{\alpha \mid 0 \leqq \alpha \leqq \gamma\} \cdots,\{\alpha \mid 0 \leqq \alpha \leqq \gamma\}$ so that, for each $\alpha<\gamma$, (a) $f(\alpha)=$ $\alpha+$ (a finite positive ordinal), and (b) Ind $\left(B_{\alpha}\right) \leqq n(f(\alpha))$. Define $f(\gamma)=$ $\gamma$ and $f(0)=\operatorname{Max}\left\{0\right.$, Ind $\left.\left(B_{0}\right)\right\}$. Assume, inductively, that $f(\delta)$ has been defined for all $\delta<\alpha<\gamma$. Then define

$$
\begin{array}{ll}
f(\alpha)=\lambda(\alpha)+\operatorname{Max}\left\{0, \operatorname{Ind}\left(B_{\alpha}\right)\right\}, & \text { if } n=0 ; \\
f(\alpha)=\operatorname{Max}\left\{\left(\lambda(\alpha)+\operatorname{Ind}\left(B_{\alpha}\right)\right),(f(\alpha-1)+1)\right\}, & \text { if } n \neq 0 .
\end{array}
$$

It is easy to check that (a) and (b) are satisfied.

Define $A_{\alpha}=B_{\lambda}$, if $\alpha=f(\lambda)$; otherwise, define $A_{\alpha}=\varnothing$. It can be verified that $X=\bigcup\left\{A_{\alpha} \mid 0 \leqq \alpha \leqq \gamma\right\}$ is a $\beta$-D-representation and that Ind $\left(A_{\alpha}\right)=-1<n(\alpha)$ or Ind $\left(A_{\alpha}\right)=$ Ind $\left(B_{f^{-1}(\alpha)}\right)$. The latter is $\leqq n(\alpha)$ by (b) above.

Theorem 3. If $X$ is the union of a locally finite collection of closed subsets each with $D$-dimension $\leqq \beta$, then $D(X) \leqq \beta$.

Proof. Let $\mathscr{C}$ be the locally finite collection of closed subsets, and, for each $C \in \mathscr{C}$, let $C=\bigcup\left\{C_{\alpha} \mid 0 \leqq \alpha \leqq \gamma(C)\right\}$ be a $\left(\gamma+\operatorname{Ind}\left(C_{\gamma}\right)\right)$ $D$-representation of $C$, such that $\left(\gamma(C)+\right.$ Ind $\left.\left(C_{\gamma}\right)\right) \leqq \beta$. If $\gamma(C)$ is less than $\delta=\lambda(\beta)$, then define $C_{\alpha}=-1$, for $\gamma(C)<\alpha \leqq \delta$. Then, using Lemma 1 , we may assume, without loss of generality, that $\gamma(C)=\delta$ and Ind $\left(C_{\delta}\right) \leqq n(\beta)$ and Ind $\left(C_{\alpha}\right) \leqq n(\alpha)$, for $\alpha<\delta$. Define $A_{\alpha}=$ $\bigcup\left\{C_{\alpha} \mid C \in \mathscr{C}\right\}$. By the Sum Theorem for metric spaces ([z], p. 17, Th. II.1) Ind $\left(A_{\alpha}\right) \leqq n(\alpha)$, for $a<\delta$, and $m \equiv \operatorname{Ind}\left(A_{\delta}\right) \leqq n(\beta)$. Thus $X=\bigcup\left\{A_{\alpha} \mid 0 \leqq \alpha \leqq \delta\right\}$ is a $(\delta+m)$-D-representation of $X$, since the locally finite union of closed sets is closed, and since each point of $X$ belongs to only finitely many $C$ 's. Therefore $D(X) \leqq \delta+m \leqq \beta$.

Corollary to Theorem 3. If $X$ is the union of two closed 
subsets $A$ and $B$, then $D(X)=\operatorname{Max}\{D(A), D(B)\}$.

THeOREM 4. If $F$ is a closed subset of the space $X$, then $D(X) \leqq$ $\lambda(D(X-F))+\operatorname{Max}\{n(D(X-F)), D(F)\} \leqq D(X-F)+D(F)$.

Proof. Let $X-F=\bigcup\left\{A_{\alpha} \mid 0 \leqq \alpha \leqq \gamma\right\}$ and $F=\bigcup\left\{B_{\alpha} \mid 0 \leqq \alpha \leqq \delta\right\}$ be $D(X-F)$ - and $D(F)$-D-representations, respectively. In the case that Ind $(F)$ is finite (and thus $\delta=0$ ), $X=\bigcup\left\{A_{\alpha} \cup F \mid 0 \leqq \alpha \leqq \gamma\right\}$ is a $\left(\gamma+\right.$ Ind $\left.\left(A_{r} \cup F\right)\right)-D$-representation of $X$, because, if $Y$ is a closed subset of $F-X$ then the closure (in $X$ ) of $Y$ is contained in $Y \cup F$. Therefore, in the case that Ind $(F)$ is finite,

$$
\begin{aligned}
D(X) & \leqq\left(\gamma+\operatorname{Ind}\left(A_{\gamma} \cup F\right)\right) \leqq\left(\gamma+\operatorname{Max}\left\{\operatorname{Ind}\left(A_{i}\right), \text { Ind }(F)\right\}\right) \\
& \leqq \lambda(D(X-F))+\operatorname{Max}\{n(D(X-F)), D(F)\} .
\end{aligned}
$$

We now assume that $\delta \geqq \omega$. Since, for each $0 \leqq n<\omega$,

$$
F-\bigcup\left\{B_{\alpha} \mid 0 \leqq \alpha \leqq n\right\}
$$

and $F-\bigcup\left\{B_{\alpha} \mid n+1 \leqq \alpha \leqq \delta\right\}$ are disjoint open subsets of $F$, and since $X$ is metrizable, there are open (in $X$ ) sets $0_{n}$ and $U_{n+1}$ such that $0_{n} \cap U_{n+1}=\varnothing, 0_{n} \cap F=F-\bigcup\left\{B_{\alpha} \mid 0 \leqq \alpha \leqq n\right\}$, and $U_{n+1} \cap F=$ $F-\bigcup\left\{B_{\alpha} \mid n+1 \leqq \alpha \leqq \gamma\right\}$. In addition, it can be seen that we may assume $0_{n} \subset 0_{n-1}, 0_{n} \subset N(F, 1 / n)=$ (the open $(1 / n)$-neighborhood of $F$ with respect to a fixed metric in $X$ ), $U_{n} \supset U_{n-1}$, and $U_{n} \supset X-$ (closure $N(F, 1 / n)$ ), for $1 \leqq n<\omega$. (Let $U_{0}=\varnothing$.) For $\alpha<\gamma$, define

$$
\begin{aligned}
C_{\alpha}= & {\left[A_{a}-N(F, 1 / n(\alpha))\right] \cup\left[\cup\left\{A_{,} \mid \lambda(\alpha) \leqq \beta<\alpha\right\}\right.} \\
& \cap(\operatorname{closure} N(F, 1 / n(\alpha-1)))-N(F, 1 / n(\alpha))] .
\end{aligned}
$$

For $0 \leqq n<\omega$, define

$$
C_{\gamma+n}=B_{n} \cup\left(A_{\curlyvee}-\left(0_{n} \cup U_{n}\right)\right) .
$$

For $\omega \leqq \beta \leqq \delta$, define $C_{\gamma+\beta}=B_{\beta}$. We shall finish the proof by showing that $X=\left\{C_{\alpha} \mid 0 \leqq \alpha \leqq \gamma+\delta\right\}$ is a

$$
\begin{aligned}
& {\left[\left(\gamma+\delta+\text { Ind }\left(C_{\hat{o}+\gamma}\right)\right)\right.} \\
& =(\lambda(D(X-F))+D(F))]-D \text {-representation of } X .
\end{aligned}
$$

For $\alpha<\gamma, C_{\alpha}$ is the finite union of closed, finite-dimensional subsets of $X-N(F, 1 / n(\alpha))$; and, thus, $C_{\alpha}$ is a closed finite-dimensional subset of $X$. For $\gamma \leqq \alpha<\gamma+\omega, C_{\alpha}$ is a subset of the union of two finite-dimensional subsets one of which is closed and thus $C_{\alpha}$ is finitedimensional; $C_{\alpha}$ is closed because it is a closed subset of $A_{\gamma} \cup F$ which in turn is closed in $X$. For $\gamma+\omega \leqq \alpha \leqq \gamma+\delta, C_{\alpha}$ is clearly closed and finite-dimensional. 
If $\beta<\gamma$, then

$$
\begin{aligned}
& \bigcup\left\{C_{\alpha} \mid \beta \leqq \alpha \leqq \gamma+\delta\right\} \\
&= \bigcup\left\{C_{\alpha} \mid \beta<\alpha<\gamma\right\} \cup \bigcup\left\{A_{\gamma}-\left(0_{n} \cup U_{n}\right) \mid 0 \leqq n<\omega\right\} \\
& \cup \bigcup\left\{B_{\tau} \mid 0 \leqq \tau \leqq \delta\right\} \\
&= \bigcup\left\{C_{\alpha} \mid \beta \leqq \alpha<\lambda(\beta)+\omega\right\} \cup \bigcup\left\{A_{\alpha} \mid \lambda(\beta)+\omega \leqq \alpha<\gamma\right\} \\
& \cup\left(A_{\gamma}-\cap\left\{0_{n} \cup U_{n} \mid 0 \leqq n<\omega\right\} \cup F\right. \\
&= \bigcup\left\{A_{\alpha} \mid \beta \leqq \alpha<\lambda(\beta)+\omega\right\} \cup\left[\bigcup\left\{A_{\alpha} \mid \lambda(\beta) \leqq \alpha<\beta\right\}\right. \\
& \cap(\operatorname{closure} N(F, 1 / n(\beta-1))] \\
& \cup \bigcup\left\{A_{\alpha} \mid \lambda(\beta)+\omega \leqq \alpha<\gamma\right\} \cup A_{r} \cup F \\
&= {\left[\bigcup\left\{A_{\alpha} \mid \lambda(\beta) \leqq \alpha<\beta\right\} \cap(\operatorname{closure} N(F, 1 / n(\beta-1))]\right.} \\
& \cup \bigcup\left\{A_{\alpha} \mid \beta \leqq \alpha \leqq \gamma\right\} \cup F,
\end{aligned}
$$

which is a closed subset of $X$. (We used here, and will use later, the fact that $(X-F) \cap \bigcap\left\{0_{n} \cup U_{n} \mid m \leqq n<\omega\right\}=V_{m} \cap(X-F)$. This is true because $U_{n} \cap 0_{n+1}$ and $0_{n} \cap U_{n+1}$ are each empty; thus all the 'cross product' terms in the expansion of $\bigcap\left\{0_{n} \cup U_{n} \mid m \leqq n \leqq p\right\}$ drop out and we are left with

$$
\begin{aligned}
& \bigcap\left\{0_{n} \cup U_{n} \mid m \leqq n \leqq p ;\right. \\
& \quad=\bigcap\left\{0_{n} \mid m \leqq n \leqq p\right\} \cup \bigcap\left\{U_{n} \mid m \leqq n \leqq p\right\}=0_{p} \cup U_{m} .
\end{aligned}
$$

Thus

$$
\begin{aligned}
\bigcap\left\{0_{n} \cup U_{n} \mid m\right. & \leqq n<\omega\}=\bigcap\left\{0_{p} \cup U_{m} \mid m \leqq p<\omega\right\} \\
& =U_{m} \cup \bigcap\left\{0_{p} \mid m \leqq p<\omega\right\}
\end{aligned}
$$

but, since $0_{n} \subset N(F, 1 / n), \bigcap\left\{0_{p} \mid m \leqq p<\omega\right\} \subset F$.)

If $\gamma \leqq \beta<\gamma+\omega$, then

$$
\bigcup\left\{C_{\alpha} \mid \beta \leqq \alpha \leqq \gamma+\delta\right\}
$$

$$
\begin{aligned}
& =\bigcup\left\{A_{\gamma}-\left(0_{n} \cup U_{n}\right) \mid n(\beta) \leqq n<\omega\right\} \cup \bigcup\left\{B_{\alpha} \mid n(\beta) \leqq \alpha \leqq \gamma+\delta\right\} \\
& \left.=\left(A_{\gamma}-\cap\left\{0_{n} \cup U_{n} \mid n(\beta) \leqq n<\omega\right\}\right) \cup \bigcup\left\{B_{\alpha} \mid n(\beta) \leqq \alpha \leqq \gamma+\delta\right)\right\} \\
& =\left(A_{\gamma}-U_{n(\beta)}\right) \cup \bigcup\left\{B_{\alpha} \mid n(\beta) \leqq \alpha \leqq \gamma+\delta\right\} \equiv\left(A_{\gamma} \cup F\right)-U_{n(\beta)},
\end{aligned}
$$

which is clearly closed. If $\gamma+\omega \leqq \beta \leqq \gamma+\delta$, then

$$
\bigcup\left\{C_{\alpha} \mid \beta \leqq \alpha \leqq \gamma+\delta\right\}=\bigcup\left\{B_{\tau} \mid \sigma \leqq \tau \leqq \delta\right\} \text {, where } \beta=\gamma+\sigma .
$$

Let $x$ be a point of $X$. We must show that there is a largest $\alpha$ such that $x \in C_{\alpha}$. If $x \in F$, then there is a largest $\beta$ such that $x \in B_{\beta}$, and thus $\gamma+\beta$ is the largest ordinal such that $x \in C_{\gamma+\beta}$. If $x \in X-F$, then there is a largest $\beta$ such that $x \in A_{\beta}$. Since $F$ is closed, $x \in X-($ closure $N(F, 1 / n)$ ), for all $n$ greater than some $N, 0 \leqq N<n<\omega$. If $\beta=\gamma$, then, for some $\gamma \leqq \alpha<\gamma+\omega, x \in C_{\alpha}$; but $x \in U_{n}$ and therefore 
$a<\gamma+n$ and thus a largest such $\alpha$ exists. If $\beta<\gamma$, then, for some $\lambda(\beta) \leqq \alpha<\lambda(\beta)+\omega, x \in C_{\alpha} ;$ but $x \notin C_{\tau}$, for $\tau>\operatorname{Max}\{\beta, \lambda(\beta)+N\}$ and therefore a largest such $\alpha$ exists.

This completes the proof of Theorem 4.

\section{Product Theorems.}

Theorem 5. For nonempty spaces $X$ and $Y$,

$$
D(X \times Y) \leqq D(X) \oplus D(Y) .
$$

(" $\oplus$ " denotes the "natural sum" of ordinal numbers. See [2], page 80; and also [10], §3, where this sum is called the upper sum. We use the convention that $\Delta \oplus \Delta=\Delta \oplus \alpha=\alpha \oplus \Delta=\Delta$. It should be noted that the natural sum is commutative, and equals the usual sum for finite ordinals.)

Proof. If either $D(X)=\Delta$ or $D(Y)=\Delta$, then the theorem holds; we therefore assume that $D(X) \neq \Delta$ and $D(Y) \neq \Delta$.

Let $X=\bigcup\left\{A_{\alpha} \mid 0 \leqq \alpha \leqq \gamma(X)\right\}$ and $Y=\bigcup\left\{B_{\alpha} \mid 0 \leqq \alpha \leqq \gamma(Y)\right\}$ be $D(X)$ - and $D(Y)$ - $D$-representations, respectively. Define

$$
C_{\alpha}=\bigcup\left\{A_{\beta} \times B_{\delta} \mid \beta \oplus \delta=\alpha\right\}, 0 \leqq \alpha \leqq \gamma(Y) .
$$

If $(x, y) \in X \times Y$, then $x \in A_{\beta}$ and $y \in B_{\tilde{\delta}}$, for some $\beta$ and $\delta$, and thus $(x, y) \in C_{\beta \oplus \delta}$. Therefore

$$
X \times Y=\bigcup\left\{C_{\alpha} \mid 0 \leqq \alpha \leqq \gamma(X) \oplus \gamma(Y)\right\} .
$$

We shall show in the next paragraph that this is a $D$-representation.

$C_{\alpha}$ is closed and finite dimensional because the equation $\beta \oplus \delta=\alpha$ has only finitely many solutions, $\beta, \delta$, and because of the product theorem for finite dimensional spaces, [7], Th.II.5. Let $\left\{\beta_{i}, \delta_{i} \mid 0 \leqq i \leqq n\right\}$ be all the ordered pairs whose natural sum is $\beta$. Then

$$
\bigcup\left\{C_{\alpha} \mid \beta \leqq \alpha \leqq \gamma(X) \oplus \gamma(Y)\right\}
$$

is equal to the closed set,

$$
\bigcup\left\{\bigcup\left\{A_{\tau} \mid \beta_{i} \leqq \tau \leqq \gamma(X)\right\} \times \bigcup\left\{B_{\tau} \mid \delta_{i} \leqq \tau \leqq \gamma(Y)\right\} \mid 0 \leqq i \leqq n\right\},
$$

because $\oplus$ is strictly increasing and, if $\tau \oplus \sigma>\beta$, then there is $\tau^{\prime} \leqq \tau$ and $\sigma^{\prime} \leqq \sigma$ such that $\tau^{\prime} \oplus \sigma^{\prime}=\beta$ (See [2], pages 80 and 81.). If $\tau$ and $\sigma$ are largest ordinals such that $x \in A_{\tau}$ and $\sigma \in A_{\sigma}$, then, since $\oplus$ is strictly increasing, $\tau \oplus \sigma$ is the largest ordinal such that $(x, y) \in C_{\tau \oplus \sigma}$.

Therefore 


$$
\begin{aligned}
D(X \times Y) & \leqq \gamma(X) \oplus \gamma(Y)+\text { Ind }\left(C_{\gamma(X) \oplus \gamma(Y)}\right) \\
& \leqq \gamma(X) \oplus \gamma(Y)+\text { Ind }\left(A_{\gamma(X)}\right)+\text { Ind }\left(B_{\gamma(Y)}\right)=D(X) \oplus D(Y),
\end{aligned}
$$

because $C_{r(X) \oplus r(Y)}=A_{r(X)} \times B_{r(Y)}$.

Theorems 6 and 7 are needed in the proof of the Product Theorem, Theorem 8.

Definition. A $D(X)$ - $D$-representation, $D=\bigcup\left\{A_{\alpha} \mid 0 \leqq \alpha \leqq \gamma\right\}$, is said to be reduced, if, for every open set $V \subset X$, such that $V \cap A_{r}$ is nonempty, it is true that $D($ closure $(V)) \geqq \gamma$.

Theorem 6. If $D(X) \neq \Delta$, then $X$ has a reduced $D(X)$-D-representation.

Proof. Let $X=\bigcup\left\{A_{\alpha} \mid 0 \leqq \alpha \leqq \gamma\right\}$ be a $D(X)$-D-representation. Let $C$ be the set of all $x \in A_{\gamma}$ such that, for some open set $V \subset X$, $x \in V$ and $D$ (closure $(V))<\gamma$. Since $X$ is paracompact, by considering the $V$ 's and the open set $X-A_{r}$, we can find a locally finite (in $X-\left(A_{r}-C\right)$ ) collection $\mathscr{Y}$ of closed (in $X$ ) sets whose interiors (in $X)$ cover $C$ but which do not intersect $A_{r}-C$ and also such that $D(W)<\gamma$, for each $W \in \mathscr{W}$.

Let $W=\bigcup\left\{A_{\alpha}(W) \mid 0 \leqq \alpha \leqq \gamma(W)<\gamma\right\}$ be a $D(W)$ - $D$-representation with the property assured in Lemma 1 . Then define, for each $\alpha \leqq \gamma$,

$$
\begin{gathered}
B_{\alpha}=\left(A_{\alpha} \text { interior }(\bigcup \mathscr{Y})\right) \cup \bigcup\left\{A_{\alpha}(W) \mid W \in \mathscr{Y} \text { and } \gamma(W)>\alpha\right\} \\
\cup\left(A_{\gamma}-C\right) \cup \bigcup\left\{A_{\gamma(W)}(W) \mid W \in \mathscr{W}\right. \text { and } \\
\left.\alpha=\gamma(W)+\operatorname{Ind}\left(A_{\gamma(W)}(W)\right)\right\} ;
\end{gathered}
$$

note that $B_{r}=A_{r}-C$. We shall show in the next paragraph (in five steps) that $X=\bigcup\left\{B_{\alpha} \mid 0 \leqq \alpha \leqq \gamma\right\}$ is a reduced $D(X)$-D-representation.

(A) The sets $\left(A_{\alpha}\right.$-interior $\left.(\bigcup \mathscr{W})\right)$ and $\left(A_{\gamma}-C\right)$ are closed in $X$, and the union of any subcollection of $\mathscr{W}$ is closed in $\left(X-\left(A_{r}-C\right)\right)$; but the boundary of $\left(C \cup\left(X-A_{r}\right)\right)$ is contained in $\left(A_{r}-C\right)$; therefore, $B_{\alpha}$ is closed in $X$. (B) By an argument similar to that in (A), we can conclude that, for each $\beta \leqq \gamma, \bigcup\left\{B_{\alpha} \mid \beta \leqq \alpha \leqq \gamma\right\}$ is closed in $X$. (C) $B_{\alpha}-\left(A_{\gamma}-C\right)$ is finite dimensional since it is the locally finite union of closed sets each of dimension less than the maximum of $\left\{\right.$ Ind $\left.\left(A_{\alpha}\right), n(\alpha)\right\}$. Therefore, $B_{\alpha}$ is finite dimensional, since each open subset of a metric space is a countable union of closed (in the whole space) sets. (D) For $x \in X$, there is a largest $\alpha$ such that $x \in B_{\alpha}$, because $x$ belongs to only finitely many $W$ 's. (E) Thus $X=$ $\mathrm{U}\left\{B_{\alpha} \mid 0 \leqq \alpha \leqq \gamma\right\}$ is a $D$-representation; and, since $B_{\gamma} \subset A_{\gamma}$, it must be a minimal representation (i.e., Ind $\left(B_{\gamma}\right)=$ Ind $\left(A_{\gamma}\right)$ ); it is reduced since 
$C$ contains all the "bad" points.

THEOREM 7. Let $A$ be a finite-dimensional, nonempty closed subset of the space $X$, and let $\gamma$ be a limit ordinal. If every open set that intersects $A$, contains a closed subset of $X$ of $D$-dimension $\geqq \gamma$, then $D(X) \geqq \gamma+$ Ind $(A)$.

Proof. Since $X$ is metric and therefore paracompact, we can find, for each $i=1,2,3, \cdots$, a locally finite collection, $\mathscr{N}_{i}$, of open subsets of $X$ each of diameter less than $1 / i$, such that, for each $i, \cup \mathscr{N}_{i}$ contains $A$ and each member of $\mathscr{N}_{i}$, intersects $A$. Then $\mathscr{N}=$ $\mathrm{U}\left\{\mathscr{N}_{i} \mid i=1,2, \cdots\right\}$ is a collection which is locally finite at points of $X-A$. For each $N \in \mathscr{N}$, let $B(N)$ be a closed (in $X$ ) set contained in $N$ and such that $D(B(N)) \geqq \gamma$. For each $N \in \mathcal{N}$, let $B(N)=$ $\bigcup\left\{A_{\alpha}(N) \mid 0 \leqq \alpha \leqq \gamma\right\}$ be a $D(B(N))$-D-representation which has the property assured by Lemma 1. (We are assuming that $D(B(N))$ is less than $\gamma+$ Ind $(A)$ because otherwise the theorem would be clearly true.) Define, for $0 \leqq \alpha \leqq \gamma$,

$$
A_{\alpha}=A \cup \cup\left\{A_{\alpha}(N) \mid N \in \mathcal{V}\right\} \text {. }
$$

We shall show in the next paragraph that $Y=\bigcup\left\{A_{\alpha} \mid 0 \leqq \alpha \leqq \gamma\right\}$ is a $D(Y)$-D-representation with $D(Y)=\gamma+\operatorname{Ind}(A)$ and that $Y$ is a closed subset of $X$. After this is done, it will follow from Theorem 1 that $D(X) \geqq \gamma+$ Ind $(A)$.

If $p \in X-Y$, then $p \notin$ closure $\left(\bigcup \mathscr{N}_{i}\right)$ for $1 / i$ less than the distance from $p$ to $A$. But outside of $\bigcup \mathscr{N}_{i}, Y=\bigcup\{B(N) \mid N \in\} \cup A$ is the union of a locally finite collection of closed sets and, thus, $Y-\cup \mathscr{N}_{i}$ is closed and $p \notin$ closure of $Y$. Therefore $Y$ is closed in $X$. For each $\beta<\gamma, \bigcup\left\{A_{\alpha} \mid \beta \leqq \alpha \leqq \gamma\right\}$ is closed, by a similar argument since it is equal to the following union of closed sets,

$$
\left.\bigcup\left\{\bigcup A_{\alpha}(N) \mid \beta \leqq \alpha \leqq \beta(N)\right\} \mid N \in \mathscr{N}\right\} \cup A \text {. }
$$

If $\alpha=\gamma+m$, where $\lambda$ is a limit ordinal and $m$ is finite, then, for each $i,(\alpha \neq \gamma) A_{\alpha}[i] \equiv \bigcup\left\{A_{\alpha}(N) \mid N \in \mathscr{V}_{i}\right\}$ is the locally finite union of closed $m$-dimensional sets and is, therefore, $m$-dimensional. (See [7], p. 17.) Thus (let Ind $(A)=n$ )

$$
A_{\alpha}=A \cup \cup\left\{A_{\alpha}[i] \mid i=1,2,3, \cdots\right\}
$$

is the union of countably many closed $m$-dimensional sets and one $n$ dimensional set; and, therefore, Ind $\left(A_{\alpha}\right)=\operatorname{Max}\{m, n\}<\infty$. By a similar argument, Ind $\left(A_{\gamma}\right)=n$. For each $x \in X$, there is a largest $\delta$ such that $x \in A_{\hat{o}}$, because either $x \in A \subset A_{\gamma}$ or $x$ belongs to only finitely many $B(N)$ 's. We have thus shown that $Y=\bigcup\left\{A_{\alpha} \mid 0 \leqq \alpha \leqq \gamma\right\}$ is 
a $(\gamma+n)$-D-representation; to show that $D(Y)=\gamma+n$, let $Y=$ $\mathrm{U}\left\{C_{\alpha} \mid 0 \leqq \alpha \leqq \delta\right\}$ be another $D$-representation of $Y$. Since $D(B(N)) \geqq \gamma$, $D(Y) \geqq \gamma$, and, therefore, $\delta \geqq \gamma$. Assume that $\delta=\gamma$ and Ind $\left(C_{\gamma}\right)<n$. Then let $p \in A_{\gamma}-C_{r}$ and let $\beta$ be the largest ordinal such that $p \in C_{\beta}$. There is an $N \in \mathscr{N}$ such that

but then

$$
p \in N \subset Y-\cup\left\{C_{\alpha} \mid \beta+1 \leqq \alpha \leqq \gamma\right\},
$$

$$
B(N)=\bigcup\left\{C_{\alpha} \cap B(N) \mid D \leqq \alpha \leqq \beta\right\}
$$

is a $D$-representation which contradicts the fact that $D(B(N)) \geqq \gamma>\beta$.

Theorem 8. $D(X \times X)=D(X)+D(Y)$, if $X$ is nonempty and has a $D(X)$-D-representation, $X=\bigcup\left\{A_{\alpha} \mid 0 \leqq \alpha \leqq \gamma\right\}$, such that either (a) $D(Y)=0$ or 1 and $A_{\gamma}$ is locally compact and nonempty or (b) $A_{r}$ and $Y$ are nonempty and $Y$ is a finite-dimensional, locally-finite polytope. (Note: If $X$ is compact then the conditions on $A_{r}$ are automatically satisfied.)

Proof. If $D(X)=\Delta$, there is nothing to prove; therefore assume that $D(X) \neq \Delta$. By Theorem 6, we may assume that the above $D(X)$ - $D$-representation of $X$ is reduced; and, since in the proof of Theorem $6 B_{\gamma}$ is a closed subset $A_{r}$ where $A_{r}$ was from any $D(X)-D$ representation, we may assume that the above representation is not only reduced but that $A_{\gamma}$ is compact. According to [6], p. 206, Ind $\left(A_{\gamma} \times Y\right)=$ Ind $\left(A_{\gamma}\right)+$ Ind $(Y)$. ("dim" = "Ind" for finite dimensional spaces.) Thus it is easily checked that

$$
X \times Y=\bigcup\left\{A_{\alpha} \times Y \mid 0 \leqq \alpha \leqq \gamma\right\}
$$

is a $(D(X)+D(Y))$ - $D$-representation. Let $N$ be any open set intersecting $A_{r} \times Y$, let $p \in N \cap\left(A_{r} \times Y\right)$, and let $y \in Y$ be the projection of $p$ in $Y$. Then $N \cap(X \times\{y\})$ is an open subset of $X \times\{y\}$ that intersects $A_{r} \times\{y\}$. Since the $D(X)-D$-representation of $X$ was reduced, there is a closed (in $X \times\{y\}$, and therefore in $X \times Y$ ) set $B \subset N \cap(X \times Y) \subset N$, such that $D(B) \geqq \gamma$. Thus by Theorem 7 ,

$$
D(X \times Y) \geqq \gamma+\text { Ind }\left(A_{\gamma}\right)+\text { Ind }(Y) \geqq D(X)+D(Y) \text {. }
$$

\section{Local property.}

Theorem 9. If $D(X)$ equals $\triangle$ or is not a limit ordinal, then there is a point $p \in X$ each of whose neighborhoods has $D$-dimension equal to $D(X)$. If $D(X)$ is a limit ordinal, then, for each $\beta<D(X)$, there is a point $p(\beta) \in X$ each of whose neighborhoods has $D$-dimension $\geqq \beta$. 
Proof. Let $X=\bigcup\left\{A_{\alpha} \mid 0 \leqq \alpha \leqq \gamma\right\}$ be a reduced $D(X)$ - $D$-representation of $X$. First, assume that $A_{\gamma}$ is not empty. Since $A_{r}$ is finitedimensional (and metrizable), there is a point $p \in A_{\gamma}$ such that each neighborhood of $p$ in $A_{r}$ has dimension equal Ind $\left(A_{\gamma}\right)$. (See [1], pp. 103,108.) We can thus conclude, using the definition of 'reduced' and Theorems 1 and 7, that each neighborhood of $p$ in $X$ has $D$-dimension equal to $D(X)$. Now suppose that $A_{r}$ is empty, or $D(X)=\Delta$, then $D(X)$ is either $\Delta$ or a limit ordinal. Let $\beta$ be any ordinal less than $D(X)$. If the Theorem failed then each point of $X$ would have a neighborhood of dimension $<\beta$. Thus, since $X$ is paracompact, we could find a locally finite closed cover of $X$ consisting of sets of $D$ dimension $<\beta$. (We need Theorem 1 here.) But in this case, Theorem 3 tells us that $D(X) \leqq \beta$, which leads to a contradiction. Since $X$ is a set and $\Delta$ is larger than all ordinal, there must be some $p \in X$ each of whose neighborhoods has $D$-dimension equal to $\Delta$, if $D(X)=\Delta$.

\section{Cardinal $(D(X))$.}

THEOREM 10. For all spaces $X$, cardinal $(D(X)) \leqq$ weight $(X)$, if $D(X) \neq \Delta$.

Proof. The theorem is clearly true for finite-dimensional spaces. Thus, let $X$ be a space such that $\omega \leqq D(X)=\alpha \neq \Delta$; and suppose, inductively, that, for all spaces, $Y$, such that $D(Y)<D(X)$, it is true that cardinal $(D(Y)) \leqq$ weight $(X)$. Let $A$ be the last $(\lambda(\alpha)$-th) member of a $D(X)$ - $D$-representation of $X . \quad D(X-A) \leqq \lambda(\alpha)$, because $X-A$ has a $\lambda(\alpha)-D$-representation. $D(X-A)=\lambda(\alpha)$, because of Theorem 4 . Then, by the definition of $\alpha$-D-representation, each point of $X-A$ has a neighborhood of $D$-dimension $<\lambda(\alpha)$; and Theorem 9 tells us that there is, for each $\beta<\lambda(\alpha)$, a point $p(\beta) \in X-A$ and an open set $N(\beta)$ containing $p$, such that $\beta \leqq D(N(\beta))<\lambda(\alpha)$. Because of the inductive assumption, we may conclude that, for each $\beta<\lambda(\alpha)$,

$$
\mathfrak{A}(\beta) \equiv \operatorname{cardinal}(D(N(\beta))) \leqq \text { weight }(N(\beta)) \leqq \text { weight }(X) .
$$

Thus the theorem is proven, if

$$
\text { cardinal }(D(X))=\text { cardinal }(\lambda(\alpha))=\operatorname{limit}\{\mathfrak{U}(\beta) \mid 0 \leqq \beta<\lambda(\alpha)\} .
$$

If this is not the case, then $\lambda(\alpha)$ must be an initial ordinal number, i.e., $\lambda(\alpha)$ is the smallest ordinal whose cardinal is cardinal $(\lambda(\alpha))$. Also, limit $\{\mathfrak{U}(\beta) \mid 0 \leqq \beta<\lambda(\alpha)\}$, if it is not equal to cardinal $(\lambda(\alpha))$, must be the immediate predecessor to cardinal $(\lambda(\alpha))$. But, then, according to [8], p. 407, $\lambda(\alpha)$ is a regular initial number and thus (see [8], p. 406) $\{D(N(\beta)) \mid 0 \leqq \beta<\lambda(\alpha)\}$ must have cardinal $(\lambda(\alpha))$ distinct 
ordinals. Thus, since we could have picked the $N(\beta)$ 's to be members of a given basis for the open sets of $X$, we conclude that

$$
\text { weight }(X) \geqq \operatorname{cardinal}(\lambda(\alpha))=\operatorname{cardinal}(D(X)) \text {. }
$$

6. Characterization of $D$-dimension. In this section we will characterize $D$-dimension from among all those topological functions (i.e., functions which are invariants of topological type) from all metric spaces to the ordinals ( $\geqq-1$ ) with $\Delta$ added. We show that any such function, $E$, which satisfies Axioms I, II, III, IV, and VII must be equal to $D$. Axioms I, II, III, IV are axioms which one would like a dimension function to satisfy. A justification of Axiom VII is contained in the next section.

I. (Finite-Dimensional Spaces). If either $E(X)$ or Ind $(X)$ are finite, then $E(X)=$ Ind $(X)$.

II. (Local Property). $E(X)=$ l.u.b. $\left\{E_{p}(X) \mid p \in X\right\}$, where $E_{p}(X)=\operatorname{minimum}\{E(N) \mid N$ a neighborhood of $p$ in $X\}$.

III. If $F$ is a closed subset of the space $X$, then $E(X) \leqq$ $E(X-F)+E(F)$.

IV. (Monotone). If $Y$ is a subspace of $X$, then $E(Y) \leqq E(X)$.

VII. If $A$ is a nonempty closed subset of $X$ and $\gamma$ is a limit ordinal, such that each open set that intersects $A$ contains, for each $\beta<\gamma$, a closed (in $X$ ) subset of $X-A$ with E-dimension $\geqq \beta$, then $E(X) \geqq \gamma+$ minimum $\{E(A), \omega\}$.

That $D$ satisfies Axioms I, II, III, IV follows easily from Theorems $1,9,4,2$, respectively. That Axiom VII is satisfied by $D$ follows from Theorem 7 and Lemma 1, below.

The proof that these axioms characterize $D$ is broken up into several parts in order to indicate where the different axioms are needed in the proof.

Lemma 1. If $D(X)<\Delta$, then $X$ contains $n$-dimensional closed subsets, for each finite $n \leqq D(X)$.

Proof. (by transfinite induction on $D(X)$ ). The lemma follows from the definition of "Ind" if $D(X)<\omega$; therefore we can assume that $D(X) \geqq \omega$. Let $X=\mathrm{U}\left\{A_{\alpha} \mid 0 \leqq \alpha \leqq \gamma\right\}$ be a $D(X)$-D-representation of $X . \quad D\left(X-A_{r}\right) \leqq \gamma$, because $X-A_{\gamma}$ has a $\gamma-D$-representation; but then Theorem 4 requires that $D\left(X-A_{\gamma}\right)=\gamma$. Let $\beta$ be an ordinal such that $n \leqq \beta<\gamma$, then by Theorem 9 there is a point $p \in X-A_{r}$ each of whose neighborhoods has $D$-dimension $\geqq \beta$. For some $\delta<\gamma$, $p \in A_{\beta}-\cup\left\{A_{\alpha} \mid \delta<\alpha \leqq \gamma\right\}$ because of the definition of $D$-representation. 
Therefore $p$ has a neighborhood, $N$, whose closure (in $X$ ) is contained in $A_{\delta}-\cup\left\{A_{\alpha} \mid \delta<\alpha \leqq \gamma\right\}$ and therefore $n \leqq \beta \leqq D$ (closure $\left.N\right) \leqq \delta<\gamma$. The lemma now follows by induction.

Theorem 11. If the function $E$ satisfies Axioms I, II, and III, then, for all spaces $X, E(X) \leqq D(X)$.

Proof. (by transfinite induction on $D(X)$ ). If $D(X)$ or $E(X)$ is finite then the theorem follows from Axiom I. Now, assume that the theorem is true for all spaces of $D$-dimension less than $\alpha$, and let $X$ be a space such that $D(X)=\alpha$. We may assume that $\omega \leqq \alpha<\Delta$ because otherwise there would be nothing to prove. Let $X=$ $\bigcup\left\{A_{\alpha} \mid 0 \leqq \alpha \leqq \gamma\right\}$ be a $D(X)$-D-representation of $X$. Each point of $X-A_{\gamma}$ has a neighborhood whose closure has $D$-dimension $<\gamma$ (see the proof of Lemma 1); thus, by the induction hypothesis, every point of $X-A_{\gamma}$ has a neighborhood whose closure has $E$-dimension $<\gamma$. Therefore, by Axiom II, $E\left(X-A_{r}\right) \leqq \gamma$. By Axiom III.

$$
E(X) \leqq E\left(X-A_{\gamma}\right)+E\left(A_{\gamma}\right) \leqq \gamma+\text { Ind }\left(A_{\gamma}\right)=D(X) \text {. }
$$

THEOREM 12. If the function $E$ satisfies Axioms I, III, IV, and VII, and if $D(X) \neq \Delta$, then $E(X) \geqq D(X)$.

Proof. (by transfinite induction on $D(X)$ ). Since $D(X) \neq \Delta, X$ has a reduced $D(X)$-D-representation, $X=\bigcup\left\{A_{\alpha} \mid 0 \leqq \alpha \leqq \gamma\right\}$, see Theorem 6 . By the definition of reduced representation, for every open set, $V$, that intersects $A_{\gamma}, D$ (closure $\left.V\right) \geqq \gamma$; and by Theorem 4, $D$ (closure $\left.(V)-A_{\gamma}\right)=\gamma$, since it has a $\gamma-D$-representation. By the definition of $D$-representation each $p \in X-A_{r}$ is contained in

$$
\bigcup\left\{A_{\alpha} \mid 0 \leqq \alpha \leqq \delta\right\},
$$

for some $\delta<\gamma$. Therefore, combining with Theorem 9, we can conclude that, for each $\beta<\gamma$ and for each $V$ as above, there is point $p \in V-A_{\gamma}$ such that $\gamma>D_{p}(V) \geqq \beta$. By Axiom IV and the induction hypothesis, the closure of each neighborhood of $p$ has $E$-dimension $\geqq \beta$. Thus Axiom VII easily applies and we obtain

$$
E(X) \geqq \gamma+E\left(A_{\gamma}\right)=\gamma+\operatorname{Ind}\left(A_{\gamma}\right)=D(X) .
$$

Lemma 2. If the function $E$ satisfies Axioms I and VII and if $X$ is an infinite-dimensional space which does not contain closed subsets of each finite dimension, then $E(X)=\Delta$.

Proof. (In [3] there is given an example of such a space $X$.) Suppose inductively that $E(Y) \geqq \beta$, for all $\beta<\alpha$, and for each infinite- 
dimensional space, $Y$, which does not contain closed subsets of each finite dimension. Note that closed infinite-dimensional subsets of such spaces have the same property. Let $X$ be one of these spaces, then $X$ must contain a closed infinite-dimensional subset, $A$, such that each open set intersecting $A$ must contain an infinite-dimensional closed (in $X$ ) subset of $X-A$. (For instance, $A$ could be an nowhere dense, infinite-dimensional closed subset of $\{x \in X \mid x$ has an infinite-dimensional neighborhood\}.) But then by Axiom VII,

$$
E(X) \geqq \lambda(\alpha)+\operatorname{minimum}\{E(A), \omega\}=\lambda(\alpha)+\omega>\alpha .
$$

THEOREM 13. If the function $E$ satisfies Axioms I, II, III, IV, and VII, and if $E(X) \neq \Delta$, then $D(X)=E(X)$.

Proof. Let $X$ be a space such that $\omega \leqq E(X)=\alpha \neq \Delta$; and suppose, inductively, that for all spaces, $Y$, such that $E(Y)<E(X)$, it is true that $E(Y)=D(Y)$. Let $A=\{x \in X \mid$ every neighborhood of $X$ has $E$ dimension $\geqq \lambda(\alpha)\}$. Clearly $A$ is closed in $X$. If $A$ failed to have closed subsets of each finite dimension, then, by Lemma $2, E(A)=\Delta$; and thus, by Axiom IV, $E(X)=\Delta$. Therefore, if Ind $(A)>n(\alpha)$, then $A$ contains a closed subset $B$, such that Ind $(B)=n(\alpha)+1$. If $V$ is an open set that intersects $B$, then $E(V) \geqq \lambda(\alpha)$ and, by Axiom III, $E(V-B) \geqq \lambda(\alpha)$. Thus, (using Axiom II applied to $V-B$ ) we conclude that $B$ and $\lambda(\alpha)$ satisfy the hypothesis of Axiom VII and we get

$$
\alpha=E(X) \geqq E(X-B)+E(B) \geqq \lambda(\alpha)+n(\alpha)+1=\alpha+1 .
$$

a contradiction. Thus it must be true that Ind $(A) \leqq n(\alpha)$. By Axiom III, $E(X-A) \geqq \lambda(\alpha)$; and thus, by Axiom II, $E(X-A)=\lambda(\alpha)$. Then, because of the definition of $A$, each point of $X-A$ has a neighborhood of $E$-dimension (and, therefore, $D$-dimension) $<\lambda(\alpha)$; and, because $E(X-A)=\lambda(\alpha)$, Axiom II tells us that there is, for each $\beta<\lambda(\alpha)$, a point $p(\beta) \in X-A$ and an open set $N(\beta)$ containing $p$, such that

$$
\beta \leqq E(N(\beta))(=D(N(\beta))<\lambda(\alpha) \text {. }
$$

Therefore, by Theorem $9, D(X-A)=\lambda(\alpha)$; and, by Theorem 4, $D(X) \leqq D(X-A)+D(A)=\lambda(\alpha)+$ Ind $(A)=\alpha=E(X)$. Theorem 13 now follows from Theorem 11 .

THEOREM 14. If the function $E$ satisfies Axioms I, II, III, IV, and VII, then $E(X)=D(X)$, for all spaces $X$.

The proof is immediate from Theorems 11,12 , and 13 .

7. Justification of Axiom VII. Axiom VII is on the surface 
quite unsatisfactory because there seems to be no obvious reason why one should require a dimension function to satisfy it. This section will give the reasons why the author is convinced that Axiom VII must be required of any satisfactory dimension function which, in addition to satisfying Axioms I-IV, also satisfies.

V. If there is a point $p \in X$, such that $E_{p}(X)=E(X)$, then $E(X \times I)=E(X)+1$. (I $\equiv$ the unit interval. $)$

VI. (Sum Theorem). If $A$ and $B$ are closed subsets of $X$, then $E(A \cup B)=\operatorname{maximum}\{E(A), E(B)\}$.

Definition $\left(Q^{\alpha}\right)$. For $0 \leqq \alpha<\omega$, define $Q^{\alpha}$ to be the finite-dimensional cell, $I^{\alpha}$. Now assume, inductively, that, for each $\beta<\alpha, Q^{\beta}$ has been defined and that $d^{\beta}$ is a metric function for $Q^{\beta}$ of bound 1 . If $\alpha=\beta+1$, then define $Q^{\beta}=Q^{\beta} \times I$ and, for $q, q^{\prime} \in Q^{\beta}$ and $t, t^{\prime} \in I$, define

$$
d^{\alpha}\left((q, t),\left(q^{\prime}, t^{\prime}\right)\right)=(1 / 2)\left[d^{\beta}\left(q, q^{\prime}\right)+\left|t-t^{\prime}\right|\right] .
$$

If $\alpha$ is an uncountable limit ordinal, then define $Q^{\alpha}$ to be the union of a point $p$ and the discrete union of all $Q^{\beta}, 0 \leqq \beta<\alpha$. Also define $d^{\alpha}(p, q)=1 /[n(\beta)+1]$, if $q \in Q^{\beta}, 0 \leqq \beta<\alpha ; d^{\alpha}\left(q, q^{\prime}\right)=d^{\beta}\left(q, q^{\prime}\right) /[n(\beta)+1]$, if both $q, q^{\prime}$ belong to $Q^{\beta}, 0 \leqq \beta<\alpha$; and $d^{\alpha}\left(q, q^{\prime}\right)=d^{\alpha}(p, q)+d^{\alpha}\left(p, q^{\prime}\right)$, if $q, q^{\prime}$ belong to different $Q^{\beta}, 0 \leqq \beta<\alpha$. If $\alpha$ is a countable limit ordinal, then define $Q^{\alpha}$ to be the one-point-compactification of the discrete union of all $Q^{\beta}, 0 \leqq \beta<\alpha$; it is easy to check that $Q^{\alpha}$ is metrizable and thus define $d^{\alpha}$ to be any (compatible) metric bounded by 1 . (For countable ordinals, this definition is due to Yu. M. Smirnov, [9], who showed that, for countable $\alpha$, Ind $\left(Q^{\alpha}\right)=\alpha$.)

THEOREM 15. If the function $E$ satisfies Axioms I-V, then $E\left(Q^{a}\right)=\alpha$.

Proof (by transfinite induction on $\alpha$ ). If $\alpha<\omega$, then Ind $\left(Q^{\alpha}\right)=$ $\alpha=E\left(Q^{\alpha}\right)$, Axiom I. Assume that, for each $\beta<\alpha, E\left(Q^{\beta}\right)=\beta$. If $\alpha$ is a limit ordinal, then $Q^{\alpha}=\{p\} \cup \bigcup\left\{Q^{\beta} \mid 0 \leqq \beta<\alpha\right\}$, where $Q^{\alpha}-\{p\}$ is a discrete union of the $Q^{\beta}, 0 \leqq \beta<\alpha$. Thus, by Axiom IV, $E\left(Q^{\alpha}-\{p\}\right) \geqq \alpha$; and, by Axiom II, $E\left\{Q^{\alpha}-\{p\} \leqq \alpha\right.$. Therefore (Axioms III and IV)

$$
\alpha=E\left(Q^{\alpha}-\{p\}\right) \leqq E\left(Q^{\alpha}\right) \leqq E\left(Q^{\alpha}-\{p\}\right)+E(\{p\})=\alpha ;
$$

and $E\left(Q^{\alpha}\right)=\alpha$. If $\alpha=\beta+1$, then (Axiom V)

$$
E\left(Q^{\alpha}\right)=E\left(Q^{\beta} \times I\right)=E\left(Q^{\beta}\right)+E(I)=\beta+1=\alpha .
$$

Definition $\left(D^{\alpha}\right)$. For $0 \leqq \alpha<\omega$, define $D^{\alpha}=I^{\alpha}$. Now assume 
inductively that $D^{\beta}$ has been defined for each $\beta<\alpha$. If $\lambda(\alpha)=\gamma$ and $n(\alpha)=n$, define $D^{\alpha}$ to be a subspace of $Q^{\alpha}=Q^{r} \times I^{n}$, such that

$$
D^{\alpha}=\left(\{p\} \times I^{n}\right) \cup \bigcup\left\{D^{\beta} \times(C(\beta))^{n} \mid 0 \leqq \beta<\gamma\right\},
$$

where

$$
\begin{aligned}
C(\beta)= & \bigcup\left\{(2 j) d^{\alpha}\left(p, Q^{\beta}\right),(2 j+1) d^{\alpha}\left(p, Q^{\beta}\right)\right] \mid j, \text { a nonnegative } \\
& \text { integer, such that } \left.(2 j+1) d^{\alpha}\left(p, Q^{\beta}\right) \leqq 1\right\} \subset[0,1]=I .
\end{aligned}
$$

THEOREM 16. If the function $E$ satisfies Axioms I-VI, then $E\left(D^{\alpha}\right)=\alpha$.

Proof (by transfinite induction on $\alpha$ ). Since $D^{\alpha} \subset Q^{\alpha}, E\left(D^{\alpha}\right) \leqq \alpha$. Assume that, for each $\beta<\alpha, E\left(D^{\beta}\right)=\beta$. If $\alpha$ is a limit ordinal, then $D^{\alpha}$ contains a copy of $D^{\beta}$ for each $\beta<\alpha$; therefore, by Axiom IV, $E\left(D^{\alpha}\right)=\alpha$. Thus, we may assume that $\lambda(\alpha)=\gamma$ and $n(\alpha)=n>0$. The theorem will follow from Axioms V and VI and the induction hypothesis, if we can show that $D^{r+n-1} \times I$ is equal to the union of 2 copies of $D^{\alpha}$. Notice that there is a map $f^{3}: C(\beta) \rightarrow[0,2]$ such that $C(\beta) \cup f^{3}(C(\beta))=[0,1+t(\beta)], f^{\beta}$ moves each point a distance of precisely $d^{\alpha}\left(p, Q^{3}\right)$, and $0 \leqq f(\beta) \leqq d^{\alpha}\left(p, Q^{\beta}\right) \leqq 1$. Since $f^{\beta}$ moves points less and less as $Q^{\beta}$ gets close to $p$, it can be shown that $D^{\alpha}$ is homeomorphic to

$$
f\left(D^{\alpha}\right) \equiv\left(\{p\} \times I^{n}\right) \cup \bigcup\left\{D^{\beta} \times(C(\beta))^{n-1} \times f^{\beta}(C(\beta)) \mid 0 \leqq \beta<\gamma\right\} ;
$$

and $D^{\alpha} \cup f\left(D^{\alpha}\right)$ is homeomorphic to

$$
\left(\{p\} \times I^{n}\right) \cup \cup\left\{D^{\beta} \times(C(\beta))^{n-1} \times[0,1+t(\beta)] \mid 0 \leqq \beta<\gamma\right\},
$$

which, because of the inequality satisfied by $t(\beta)$, is homeomorphic to $D^{r+n-1} \times I$.

REMARK. For countable $\alpha$, Ind $\left(Q^{\alpha}\right)=\alpha$; but, for uncountable $\alpha$, Ind $\left(Q^{\alpha}\right)$ does not exist because, for these $\alpha, Q^{\alpha}$ is not weakly infinitedimensional. (See [7], p. 177.) Also, it can be shown that Ind $\left(D^{\alpha}\right)=\omega$, for all countable $\alpha$. This shows that the Sum Theorem does not hold for Ind, because

$$
D^{\omega+1} \cup f\left(D^{\omega+1}\right)=D^{\omega} \times I=Q^{\omega} \times I=Q^{\omega+1} .
$$

(This is essentially the same as the example given by B. T. Levšenko, [5], p. 258.)

Let $X$ be a space which is the disjoint union of a closed 1-dimensional set $A$ and the members of a collection of closed sets, $\mathscr{F}$, such that each open set which intersects $A$ must contain, for each 
$n<\omega$, a member of $\mathscr{F}$ of dimension equal to $n$. Even if $X$ is compact it seems very unlikely that Axioms I-VI will, in general, determine the dimension of $X$. Also, it seems clear that any satisfactory dimension would have to assign the same dimension to $X$ as it does to $D^{\omega+1}$ (which has the same structure as $X$ ), for if it didn't it would be a dimension theory that distinguished between the dimensional-properties of $I^{n}$ and an $n$-dimensional space. Thus, Axiom VII follows from Axioms I-VI (through Theorem 16) and the requirement that if $X$ is put together like $D^{\alpha}$ with $\geqq n$-dimensional sets replacing $n$-cells of $D^{\alpha}$, then $E(X) \geqq E\left(D^{\alpha}\right)$.

REMARK. Axioms I-VI do not characterize $D$ for all separable metric spaces. To see this, define $E(X)=D(X)=\operatorname{Ind}(X)$, if $X$ is finite-dimensional, otherwise define $E(X)=$ l.u.b. $[\{\omega\} \cup\{D(Y) \mid Y \subset X$ and $Y$ is compact $\}^{1}$. It can be checked that $E$ satisfies Axioms I-VI for all separable metric space. (The hypothesis of separability is needed to verify Axiom V.) Let $R$ be a subspace of $D^{\omega+2}$ which is obtained by replacing $\left(\{p\} \times I^{2}\right)$ by a subset which is 1-dimensional, separable, and totally disconnected (see, for example, [4], p. 22). Then $D(R)=\omega+1$ but $E(R)=\omega$.

The author wishes to thank the referee for suggestions which have improved the presentation in this paper.

\section{REFERENCES}

1. C. H. Dowker, Local dimension of normal spaces, Quart. J. Math., Oxford, 6 (1955), 101-120.

2. F. Hausdorff, Set Theory, Second Edition, Chelsea Publishing Co., New York, 1962.

3. D. W. Henderson, An infinite-dimension compactum with no positive-dimensional compact subsets-A simpler construction, Amer. J. Math. 89 (1967), 105-121.

4. W. Hurewicz and H. Wallman, Dimension Theory, Princeton University Press, 1941.

5. B. T. Levšenko, Spaces with transfinite dimension (Russian), Mat. Sb. (N. S.)(109) 67 (1965), 255-266. (Б. Т. Левшенко, Мат. Сб., Н. С.)

6. K. Morita, On the dimension of product spaces, Amer. J. Math. 75 (1953), 205223.

7. J. Nagata, Modern Dimension Theory, P. Noordhoff N. V., Groningen, (and John Wiley \& Sons, Inc., New York) 1965.

8. W. Sierpinski, Cardinal and Ordinal Numbers, Second Edition, PWN, Warszawa, 1965.

9. Yu. M. Smirnov, On universal spaces for certain classes of infinite-dimensional spaces, Amer. Math. Soc. Translations (2) 21 (1962), 21-34. [translated from Ю. М. Смирнов, Изв. Акад. Наук СССР, Сер. Мат. 23 (1959), 185-196].

10. G. H. Toulmin, Shuffing ordinals and transfinite dimension, Proc. London Math. Soc. (3) 4 (1954), 177-195.

1 This function was kindly suggested by the referee. 
Received July 26, 1967 and in revised form October 25, 1967. The author was partially supported by NSF Grant GP-3685.

CoRnell UNIVERSITY

ITHACA NeW YoRK 



\section{PACIFIC JOURNAL OF MATHEMATICS}

\section{EDITORS}

\author{
H. ROYDEN \\ Stanford University \\ Stanford, California \\ R. R. Phelps \\ University of Washington \\ Seattle, Washington 98105
}

J. DUGUNDJI

Department of Mathematics

University of Southern California

Los Angeles, California 90007

RICHARD ARENS

University of California

Los Angeles, California 90024

\section{ASSOCIATE EDITORS}
E. F. BECKENBACH
B. H. NeumanN
F. WOLF
K. YosidA

\section{SUPPORTING INSTITUTIONS}

UNIVERSITY OF BRITISH COLUMBIA

CALIFORNIA INSTITUTE OF TECHNOLOGY

UNIVERSITY OF CALIFORNIA

MONTANA STATE UNIVERSITY

UNIVERSITY OF NEVADA

NEW MEXICO STATE UNIVERSITY

OREGON STATE UNIVERSITY

UNIVERSITY OF OREGON

OSAKA UNIVERSITY

UNIVERSITY OF SOUTHERN CALIFORNIA
STANFORD UNIVERSITY

UNIVERSITY OF TOKYO

UNIVERSITY OF UTAH

WASHINGTON STATE UNIVERSITY

UNIVERSITY OF WASHINGTON

AMERICAN MATHEMATICAL SOCIETY CHEVRON RESEARCH CORPORATION TRW SYSTEMS

NAVAL WEAPONS CENTER

Printed in Japan by International Academic Printing Co., Ltd., Tokyo, Japan 


\section{Pacific Journal of Mathematics}

\section{Vol. 26, No. $1 \quad$ November, 1968}

Efraim Pacillas Armendariz, Closure properties in radical theory......... 1

Friedrich-Wilhelm Bauer, Postnikov-decompositions of functors .......... 9

Thomas $\mathrm{Ru}-$ Wen Chow, The equivalence of group invariant positive definite

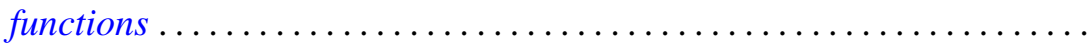

Thomas Allan Cootz, A maximum principle and geometric properties of

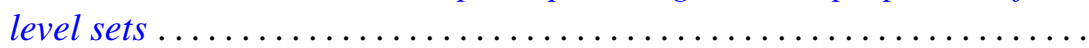

Rodolfo DeSapio, Almost diffeomorphisms of manifolds ............ 47

R. L. Duncan, Some continuity properties of the Schnirelmann density......

Ralph Jasper Faudree, Jr., Automorphism groups of finite subgroups of

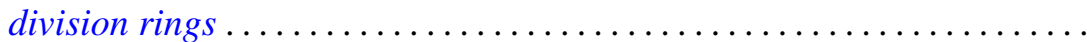

Thomas Alastair Gillespie, An invariant subspace theorem of $J$.

Feldman.........................................

George Isaac Glauberman and John Griggs Thompson, Weakly closed direct factors of Sylow subgroups .............................

Hiroshi Haruki, On inequalities generalizing a Pythagorean functional equation and Jensen's functional equation .....................

David Wilson Henderson, D-dimension. I. A new transfinite dimension.....

David Wilson Henderson, D-dimension. II. Separable spaces and

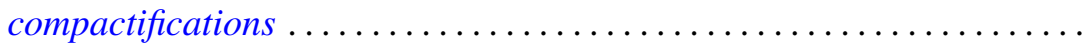

Julien O. Hennefeld, A note on the Arens products ............... 115

Richard Vincent Kadison, Strong continuity of operator functions ...

J. G. Kalbfleisch and Ralph Gordon Stanton, Maximal and minimal coverings of $(k-1)$-tuples by $k$-tuples.

Franklin Lowenthal, On generating subgroups of the Moebius group by pairs of infinitesimal transformations...

Michael Barry Marcus, Gaussian processes with stationary increments possessing discontinuous sample paths . .

Zalman Rubinstein, On a problem of Ilyeff ...

Bernard Russo, Unimodular contractions in Hilbert space. ...

David Lee Skoug, Generalized Ilstow and Feynman integrals...

William Charles Waterhouse, Dual groups of vector spaces . 